\title{
Análise comparativa entre arranjos de tomografia elétrica no reconhecimento de estruturas de fluxo em aquífero fraturado em Caçapava do Sul (RS)
}

\author{
César Augusto MOREIRA', Lívia Portes Innocenti HELENE², Fernanda Teles Gomes \\ ROSA², Marco Antônio Fontoura HANSEN³, Walter MALAGUTTI FILHO'1, João Carlos \\ DOURADO' \\ 1 Departamento de Geologia Aplicada, Universidade Estadual Paulista. Av. 24-A, 1515, Bela Vista, CEP \\ 13506-900,Rio Claro,SP, Brasil (moreirac@rc.unesp.br, malaguti@rc.unesp.br, dourado@rc.unesp.br). \\ 2 Programa de Pós-Graduação em Geociências e Meio Ambiente, Universidade Estadual Paulista. \\ Av. 24-A, 1515, Bela Vista, CEP 13506-900, Rio Claro, SP Brasil \\ (liviapihelene@gmail.com, fernandatgr1@gmail.com). \\ ${ }^{3}$ Universidade Federal do Pampa, Av. Pedro Anunciação, s/n, Vila Batista, 96570-000 Caçapava do Sul, \\ RS, Brasil (marcohansen@unipampa.edu.br).
}

\begin{abstract}
Resumo. Este trabalho apresenta uma análise comparativa entre dados de tomografia elétrica de detalhe, obtidos sobre terreno granítico onde ocorre fluxo subterrâneo em sistema aquífero fraturado, adquiridos por meio dos arranjos dipolo-dipolo, Schlumberger e Wenner, na região de Caçapava do Sul (RS). Dados estruturais obtidos em exposições de rochas entre $100 \mathrm{~m}$ e $300 \mathrm{~m}$ da área de estudo serviram de base para análise dos produtos geofísicos e definição do modelo matemático mais factível a realidade geológica. O fraturamento predominante na região possui orientação E/W com dois sentidos principais de mergulho. Nos três arranjos de aquisição tomográfica analisados, foi possível o reconhecimento de zonas de baixa resistividade relacionadas ao acúmulo de água, com valores inferiores a $70 \Omega$.m, em contraste com valores superiores a $1000 \Omega$.m típicos de rocha inalterada. Os resultados referentes ao arranjo Schlumberger permitiram o modelamento 3D de uma superfície de baixa resistividade com orientação e mergulho compatíveis com sistemas de fraturas reconhecidos em afloramentos, em detrimento aos demais resultados, que não possibilitaram subsídios para uma definição adequada de locais para perfuração de poços.
\end{abstract}

Palavras-chave. aquífero fraturado, geofísica, tomografia elétrica, dipolo-dipolo, Schlumberger, Wenner

\begin{abstract}
COMPARATIVE ANALYSIS AMONG ELETRICAL RESISTIVITY TOMOGRAPHY ARRAYS IN THE RECONNAISSANCE OF FLOW STRUCTURE IN FRACTURES AQUIFER IN CAÇAPAVA DO SUL (RS). ThiS paper performs presents a comparative analysis between data from detailed electrical resistivity tomography data acquired through the dipole-dipole, Schlumberger and Wenner arrays of detail, obtained on a ground where granite terrain in Caçapava do Sul (RS) where groundwater flows in occurs underground flow in a fractured aquifer system, acquired through the arrangements make dipole-dipole, Schlumberger and Wenner. Structural data obtained on rocks exposed in $100 \mathrm{~m}$ and $300 \mathrm{~m}$ offrom the study area served as a fundamental the basis for analysisthe analysis of geophysical products and the definition of the mathematical model that is more feasible with the reality. The predominant fracture direction in the region has E/W orientation with two main senses of diving. By the results of the three arrays, it was possible to recognize areas of low resistivity related to water accumulation, with values lower than $70 \Omega$.m, in contrast to values higher than $1000 \Omega$.m typical of unaltered rock. The results of the arrangement allowed Schlumberger array allowed a 3D modeling a surface of low resistivity surface with orientation and dive compatible with the fracture system recognized in field, in this way this array showed a more realist model guidance and diving are compatible with systems of fractures found in rock, to the of the geological structures in detriment to the other results, which do not allow subsidies for an adequate definition of sites for drilling wells.
\end{abstract}

Keywords. fractured aquifer, geophysics, electrical resistivity tomography, dipole-dipole, Schlumberger, Wenner 


\section{Introdução}

Aquíferos fraturados são sistemas de águas subterrâneas particularmente desafiadores quanto à definição de áreas favoráveis a perfuração de poços de alta vazão. A complexidade estrutural intrínseca de fluxo de água em rochas cristalinas frequentemente resulta no predomínio de poços secos ou com vazões insuficientes ao atendimento das demandas sociais. Contudo, em muitos casos, os aquíferos fraturados podem ser utilizados de forma racional a partir da integração de estudos hidrogeológicos, análises estruturais e levantamentos geofísicos.

O uso de métodos geofísicos é uma possibilidade racional neste contexto, diante de fatores como sensibilidade de mensuração indireta de parâmetros físicos alteráveis em presença de águas em fraturas, ampla cobertura para investigação em termos espaciais e versatilidade de investigações em profundidades variáveis em prazos relativamente reduzidos. 0 aspecto essencial de estudos geofísicos em sistemas aquíferos é a redução de incertezas e a possibilidade de definição de alvos promissores para captação, e a consequente redução de custos com perfurações de poços secos (Sara, 2003; Knödel et al., 2007).

Os métodos geofísicos elétricos e eletromagnéticos são particularmente relevantes em estudos hidrogeológicos diante da sensibilidade do instrumental na detecção de variações no teor de umidade nos materiais geológicos, fator que influencia diretamente parâmetros físicos como resistividade ou condutividade elétrica (Rubin \& Hubbard, 2005; Milsom \& Eriksen, 2011). A eficácia destas ferramentas de investigação do ambiente geológico em aquíferos com influência de cunhas salinas, arquitetura estratigráfica, estudos de vulnerabilidade ou diagnóstico de contaminações em aquíferos é demonstrada em diversos trabalhos (Salem, 2001; Zhou et al., 2001; Chambers et al., 2006; Sequeira Gómez et al., 2010; Dena et al., 2012; Arango-Galván et al., 2016; Helene et al., 2016; Moreira et al., 2017).

A aplicação de estudos de tomografia elétrica (ERT) possibilita análises bidimensionais em resolução adequada, com densidade amostral e profundidades variáveis que podem revelar zonas fraturadas com potencial aquífero. Um paradigma particularmente desafiador é a adaptação de um método de investigação com formulação matemática baseada em meios isotrópicos e homogêneos para uso em estruturas altamente anisotrópicas e heterogêneas (Orellana, 1972; Telford et al., 2004). Neste sentido, a análise de arranjos de aquisição de dados e das interações do campo elétrico e potencial em estruturas geológicas complexas, pode ser a chave para reconhecimentos de limitações e de viabilidade de procedimentos mais adequados ao estudo de sistemas aquíferos fraturados, com o objetivo de locação de pontos favoráveis a perfuração de poços produtores.

Neste sentido, o presente trabalho apresenta e discute os resultados de aplicação do método geofísico da Eletrorresistividade em estudo de sistema aquífero fraturado contido em granito, onde foram realizados ensaios de tomografia elétrica (ERT) com os arranjos dipolo-dipolo, Wenner e Schlumberger, estes definidos a título de comparação, quanto a maior aplicabilidade em diversos trabalhos publicados, e pertinentes discussões quanto as resoluções horizontais e verticais (Gandolfo \& Gallas, 2005; Moreira et al., 2016), além de análise estrutural na rocha para definição dos principais grupos de fraturas. O principal objetivo é uma análise do arranjo mais adequado em termos de sensibilidade e resolução, que resulte em um produto de modelamento matemático condizente com a realidade geológica local e possibilite a seleção de alvos para perfuração e captação de água subterrânea.

Optamos aqui pelo processamento básico de um programa utilizado de forma dominante no Brasil para processamento de dados geoelétricos 2D (Res2Dinv) com vistas a uma análise comparativa de arranjos focados no estudo de aquífero fissural. Esta premissa eventualmente gerou artifícios de processamento por não haver tratamento individual personalizado do produto de cada arranjo, mas possibilitou um resultado próximo da realidade (ou podemos dizer mais técnico), que de forma objetiva aponta vantagens e desvantagens de emprego de cada arranjo 
neste tipo de aquífero.

\section{2 Área, materiais e métodos}

\section{1 Localização da área}

A área de estudo está localizada numa propriedade rural distante $6 \mathrm{Km}$ a norte do centro da cidade de Caçapava do Sul, município localizado na região centro-sul do Estado do Rio Grande do Sul (Fig. 1). Esta região do Estado é constituída por municípios de pequeno porte e com população variável entre 10.000 a 60.000 habitantes. A atividade econômica é baseada na agricultura da soja, pecuária extensiva e serviços comerciais básicos.
Os serviços de saneamento básico são geridos pela Corsan (Companhia Riograndense de Saneamento S/A), empresa estatal estadual criada em 1965 para atendimento a demandas de abastecimento público, que atualmente atende $2 / 3$ da população estadual ou 6 milhões de pessoas em 316 municípios do Rio Grande do Sul.

A área urbana dos municípios sob sua administração é quase universalmente servida por fornecimento intermitente de água potável, embora apresente índices abaixo de 30\% de coleta e esgotamento sanitário. A área rural é predominantemente servida por captação superficial ou subterrânea, independente da rede pública, que serve somente os centros urbanos.

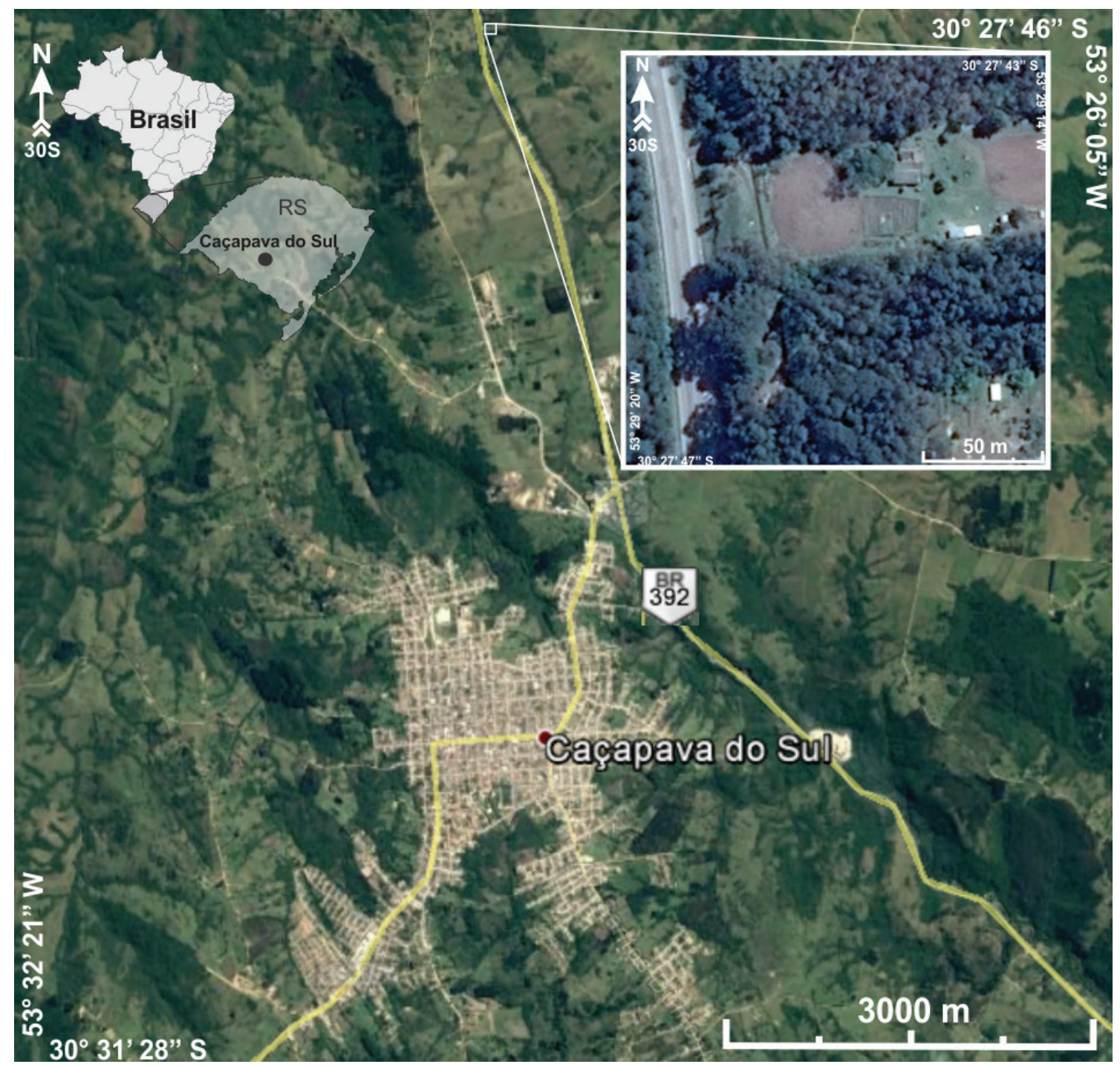

Figura 1. Localização da área de estudo.

Figure 1. Localization of the study area. 


\subsection{Contexto geológico}

Esta área integra parte da Suíte Granítica Caçapava do Sul, classificada como granitóides sintranscorrentes contidos no Domínio Ocidental do Escudo Sul-Rio-Grandense (CPRM, 2000). Os granitóides sintranscorrentes são representados por corpos plutônicos intrusivos nas unidades metamórficas do Domínio Ocidental, com foliação milonítica relacionada a eventos transcorrentes.

A Suíte Granítica Caçapava do Sul compreende um corpo com formato aproximadamente dômico, com cerca de 25 $\mathrm{Km}$ de extensão e orientado na direção N/S, totalmente circundado por rochas metamórficas de baixo grau em contato abrupto. No contato das rochas graníticas com as encaixantes do Complexo Metamórfico Vacacaí - unidade metassedimentar, ocorrem xenólitos da encaixante na intrusiva, além de apófises, geralmente concordantes ou de baixo ângulo com a foliação principal dos metamorfitos. A foliação milonítica que afeta os granitoides é de maneira geral concordante com a foliação principal da sequência metamórfica.

Esta suíte é constituída dominantemente por monzo e sienogranitos, com participação subordinada de rochas de composição granodiorítica a tonalítica presentes na borda oeste do corpo principal. Devido aos diversos tipos petrográficos, é composta por três fácies principais: biotita granitos, leucogranitos e granitos transicionais (CPRM, 2000).

A constituição mineralógica principal está representada por proporções variadas de quartzo, feldspato alcalino e plagioclásio, com biotita presente nas diversas fácies $\mathrm{e}$ hornblenda identificada apenas nos tipos tonalíticos. Os minerais acessórios mais comuns são representados por zircão, allanita, apatita e minerais opacos, além de titanita e rutilo como acessórios. Clorita, epidoto, muscovita, carbonato e opacos secundários constituem a mineralogia resultante do metamorfismo associado à fase de deformação transcorrente (CPRM, 2000).

Os solos correspondentes à área da suíte granítica são classificados em duas unidades: argissolo vermelho-amarelo eutrófico e argissolo vermelho-amarelo distrófico (EMBRAPA, 2018).
As rochas da suíte granítica afloram sob a forma de blocos ou em extensas superfícies em vales de drenagens, com acentuada foliação milonítica orientada segundo NNE/SWS e mergulho vertical, marcada pela orientação de minerais máficos e estiramento de cristais de quartzo e feldspato. Estreitas faixas de milonitos cinza-escuro alternadas com feições de textura protomilonítica, definem as zonas de maior deformação durante o resfriamento e cristalização, onde são reconhecidos porfiroclastos de feldspato rotacionados e sombras de pressão.

Análises estruturais realizadas por Bittencourt (1983) nas regiões norte e sudeste da suíte granítica permitiram o reconhecimento de três fases de deformação: (I) fase em porfiroblastos pré-cinemáticos em relação à xistosidade regional das encaixantes, (II) fase relacionada à xistosidade regional que também afetou os granitos e (III) fase geradora de estrutura antiformal com posicionamento de suíte granítica em seu núcleo central. Deste conjunto, as duas últimas fases de deformação afetaram de forma expressiva a suíte granítica, regionalmente definida como fases D3 e D4, resultado da vigência de esforços compressivos durante o resfriamento do corpo intrusivo.

Estruturas de deformação rúptil são as mais relevantes aos estudos hidrogeológicos no âmbito da Suíte Granítica Caçapava do Sul, que constitui um sistema aquífero fraturado potencialmente utilizável para fins de abastecimento, além de aquífero livre na interface de contato solo/rocha.

\subsection{Materiais e métodos}

Os estudos se iniciaram com as análises geológicas estruturais em exposições de granitos nas proximidades da área de estudo, para definição das principais famílias de fraturas e posterior programação de ensaios geofísicos. A rodovia de acesso à área de estudo apresenta algumas exposições de rocha pouco intemperizada, com estruturas claramente reconhecíveis com mergulho alto e médio ângulo $\left(90^{\circ}\right.$ a $\left.42^{\circ}\right)$ e orientação geral entre $N 270^{\circ}$ e N280 (Fig. 2A e 2B). 
A aquisição de dados geofísicos consistiu na realização de leituras de resistividade elétrica por aplicação da técnica de tomografia de resistividade elétrica (ERT), em 4 linhas de investigação com comprimento individual de $105 \mathrm{~m}$ e espaçamento entre eletrodos de $5 \mathrm{~m}$, dispostas paralelamente e separadas a cada 5 m, orientadas na direção $N 350^{\circ}$, basicamente perpendiculares a orientações das potenciais estruturas de fluxo de água subterrânea (Fig. 3).

As linhas de tomografia elétrica foram realizadas a partir dos arranjos dipolo-dipolo, Wenner e Schlumberger, para avaliação comparativa da sensibilidade individual e possibilidade de reconhecimento e modelamento de fraturas com potencial de fluxo de água subterrânea para captação por poço.

O equipamento geofísico utilizado foi o resistivímetro Terrameter LS, fabricado pela ABEM Instrument (Suécia), que consiste em módulo único de transmissão e recepção de sinais automatizado a partir de programação prévia, com potência de $250 \mathrm{~W}$, resolução de $1 \mu \mathrm{V}$ e corrente máxima de 2,5 A. Permite a realização de ensaios de potencial espontâneo (SP), eletrorresistividade (ER) e polarização induzida (IP) por meio de ciclos periódicos de transmissão e recepção de sinais, cálculo automático da resistência de contato e do desvio padrão do conjunto de medidas.
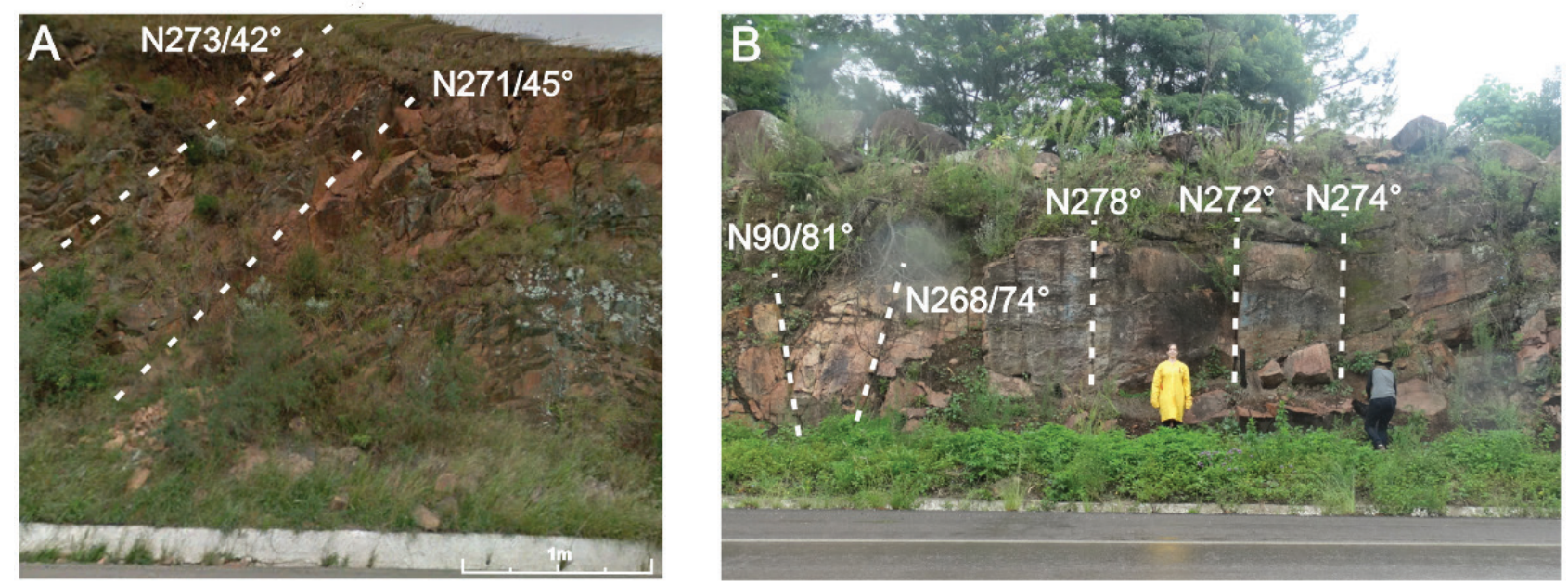

Figura 2. Orientação estrutural em granito exposta em rodovia de acesso a área de estudo. A) Fraturas de médio ângulo de mergulho; B) Fraturas verticais.

Figure 2. Structural orientation in exposed granites in highway access in the study area. A) Fractures of medium angle of dip; B) Vertical fractures.

As medidas adquiridas em campo foram modeladas no programa Res2dinv e resultaram em seções de resistividade em termos de distância x profundidade, com escala gráfica logarítmica e intervalos de interpolação de valores em cores. Este é um programa que determina automaticamente um modelo bidimensional de subsuperfície, a partir de dados de resistividade ou cargabilidade obtidos em ensaios de caminhamento elétrico (Griffiths \& Barker, 1993).

O modelo 2D utilizado no programa divide a pseudo-seção em blocos retangulares, que representarão a pseudo-seção pelo ajuste das medidas de campo. Esta otimização procura reduzir a diferença entre os valores de resistividade aparente, calculados e medidos em campo, pelo ajuste da resistividade do modelo de blocos, cuja diferença é expressa pelo erro RMS (Root Mean Squared) e seu produto é representado sob forma de modelos de inversão (Loke \& Barker, 1996).

O produto numérico de inversão bidimensional dos dados de cada seção foi reunido em planilha única, que contempla a posição das leituras ao longo das linhas (variável " $x$ "), espaçamento entre linhas (variável " $y$ "), profundidade modelada pela inversão (variável "z") e o valor de resistividade elétrica (variável " $R$ ").

Esta planilha foi utilizada para geração de modelos de visualização 3D, numa rotina de etapas básicas adotadas em pesquisa 
mineral. Neste caso, o plano de amostragem é frequentemente definido a partir de critérios estatísticos, estruturais, disposição espacial de uma acumulação mineral, dentre outros (Moon et al., 2006). Um procedimento simples consiste em amostragens por um conjunto de furos perpendicular ao eixo principal da estrutura, sucedido por um conjunto paralelo de linhas de furo.

A resolução da malha de amostragem é condicionada ao espaçamento entre furos, entre linhas de furos e entre quantidade de amostras coletadas por furo. De qualquer forma, o resultado analítico das amostras é tabelado e modelado em termos bidimensionais e posteriormente interpolado em termos tridimensionais. Cada ponto do modelo 3D final é transformado num bloco, com dimensões condicionadas a critérios estatísticos e de malha amostral, ao qual é atribuído um teor baseado em análises químicas e um valor médio de densidade relativo à rocha que hospeda o minério. A relação entre teor em volume possibilita o cálculo de reservas e a viabilidade econômica do empreendimento (Moon et al., 2006).

Modelos geofísicos de visualização 3D derivados de seções 2D, também conhecidos com modelos quasi-3D ou pseudo-3D, proporcionam uma compreensão bastante ampla da complexidade de estruturas geológicas, modelamento de litotipos ou depósitos minerais (Chambers et al., 2006; Aizebeokhai et al., 2011; Moreira et al., 2012; Moreira et al., 2016; Vieira et al., 2016; Côrtes et al., 2016).

\section{Resultados}

Os modelos de inversão de resistividade representativos da área são apresentados em escala única e logarítmica de valores, que variam

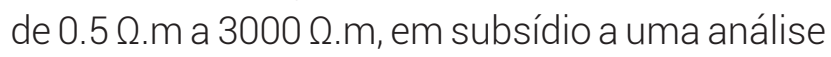
comparativa entre os arranjos de aquisição

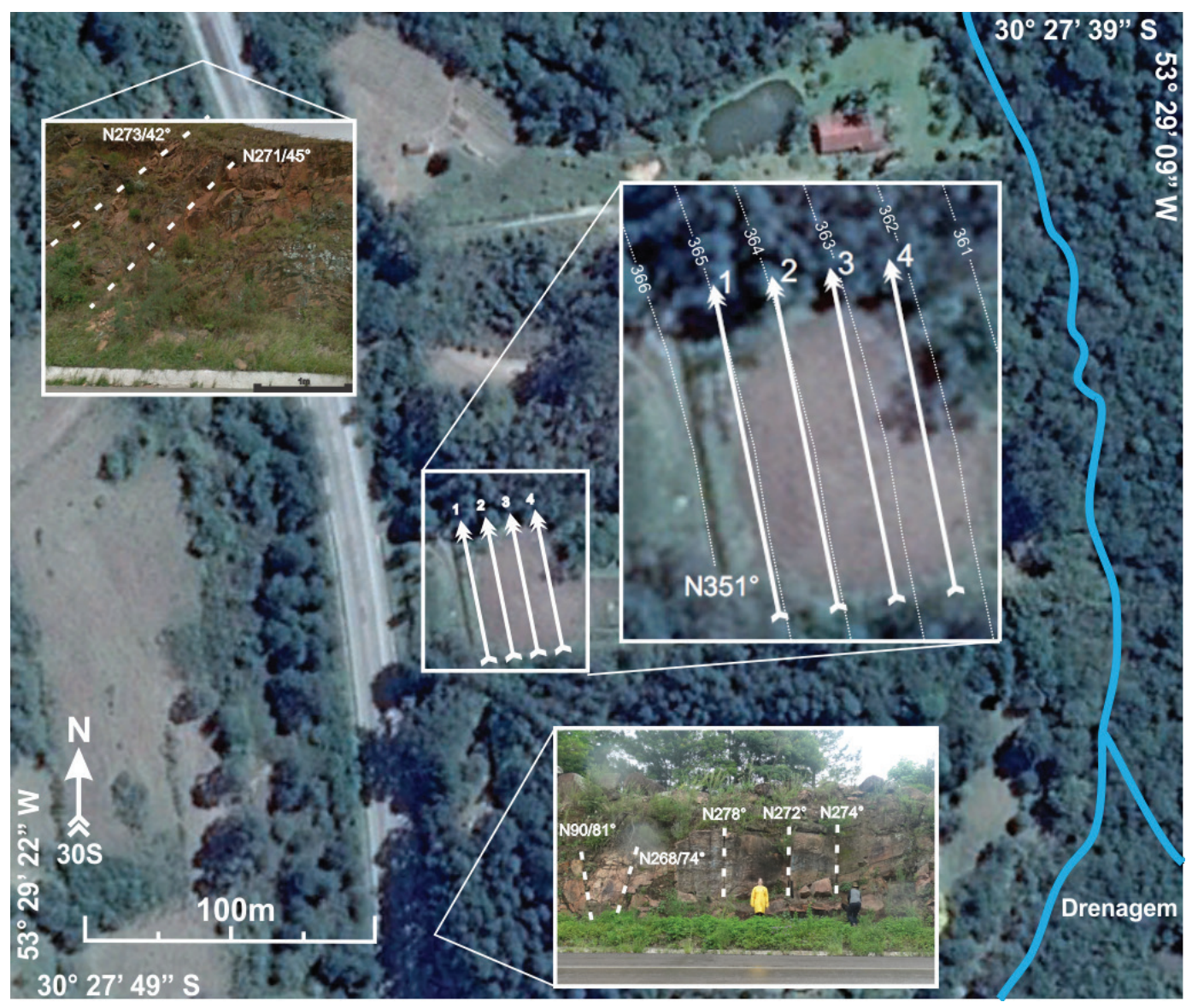

Figura 3. Imagem aérea da área de pesquisa com o posicionamento e orientação das linhas de tomografia de resistividade elétrica em relação ao fraturamento e ao relevo local.

Figure 3. Aerial image of the research area with the positioning and of electrical resistivity tomography lines in relation of fractures and local relief. 
tomográfica. As seções processadas resultaram em modelos de inversão para até $9.56 \mathrm{~m}$ de profundidade. 0 parâmetro RMS é bastante alto em todos os modelos e pode ser atribuído a ampla faixa de variação entre os dados ou extremos ao redor da média, com elevados valores associados ao granito inalterado e baixos relacionados a solos argilosos saturados ou zonas de acúmulo de água.

O reconhecimento geológico em campo realizado durante a etapa de aquisição de dados estruturais permitiu a identificação de solo saprolítico com espessura entre 0,3 m a 1,2 m. Neste sentido, os modelos de inversão refletem uma realidade geológica basicamente representada por granitos inalterados, eventuais descontinuidades estruturais e níveis argilosos saturados.

Em termos gerais, os modelos de inversão apresentados a partir dos três arranjos, são caracterizados por uma faixa central de alta resistividade, números superiores a $100 \Omega$.m até cerca de $3000 \Omega . m$, de acordo com a escala determinada para comparação entre os arranjos, esta ampla variação corresponde ao grau de saturação por água no granito, quanto maiores os valores obtidos de resistividade para esta litologia, menor o indicativo de saturação por água e intemperismo, acompanhados por faixas de baixa resistividade, de 0,500 $\Omega$.m a $100 \Omega . m$, nos extremos iniciais e a partir de $25 \mathrm{~m}$ até o final dos modelos, com particularidades específicas para cada arranjo (Fig. 4A e 4B).

Os dados para o arranjo dipolo-dipolo resultaram em modelos robustos ou pouco suavizados, embora sejam baseados numa elevada densidade de amostras por unidade de área (96 medidas por seção). A zona de baixa resistividade inicial é bastante restrita e poderia ser atribuída a efeitos de borda resultantes do processamento. A zona de baixa resistividade final é rasa e restrita ao máximo de $4 \mathrm{~m}$ de profundidade, espessura bastante superior a uma eventual camada de solo saturado (Fig. 4A e 4B).

Os dados para o arranjo Schlumberger resultaram nos modelos com maior suavização e maior densidade amostral por unidade de área (102 medidas por seção), com uma clara definição de uma zona de baixa resistividade inicial com mergulho desde a superfície até a base das seções. A zona de baixa resistividade a partir de $25 \mathrm{~m}$ atinge até $7,5 \mathrm{~m}$ de profundidade, onde predominam valores inferiores a $6 \mathrm{~m}$ típicos de material argiloso totalmente saturado, possivelmente resultante de uma faixa de rochas inicialmente bastante fraturadas e fortemente intemperizadas (Fig. 4A e 4B).

Os dados para o arranjo Wenner resultaram em modelos relativamente suavizados mesmo com uma baixa densidade amostral (42 medidas por seção). Em termos gerais existe algo semelhante ao descrito para os dados do arranjo Schlumberger, com duas zonas de baixa resistividade nos extremos inicial e final das seções. 0 extremo inicial de baixa resistividade é contínuo desde a superfície até $7,5 \mathrm{~m}$ de profundidade, ao passo que a zona de baixa resistividade do terço final das seções é limitada a 5,5 m de profundidade (Fig. 4A e 4B).

A interpolação dos modelos de inversão e a geração de blocos de visualização 3D com ajuste da topografia local permite uma análise integrada de continuidade das zonas de baixa resistividade. Nos blocos para os três arranjos é visível a continuidade de duas zonas de baixa resistividade em posições coincidentes, com pequenas diferenças quanto ao predomínio de valores e formatos (Fig. 5).

A zona de baixa resistividade no extremo final das seções no modelo dipolo-dipolo varia de $5 \mathrm{~m}$ a $10 \mathrm{~m}$ de largura, enquanto a zona de baixa resistividade do extremo inicial do bloco é limitada entre $2 \mathrm{~m}$ a $5 \mathrm{~m}$ de profundidade, e ambas cruzam todo o modelo na direção E/W. No modelo de blocos 3D para o arranjo Schlumberger a zona do extremo final apresenta valores relativamente mais elevados e atenuados em relação aos demais modelos, enquanto a zona do extremo inicial do bloco mostra continuidade em profundidade, com mergulho médio de $40^{\circ}$ no sentido norte. O modelo de blocos 3D para o arranjo Wenner exibe uma zona de baixa resistividade no final do bloco com formato retilíneo e bastante definido, ao passo que a zona do extremo inicial do bloco também é retilínea, posicionada abaixo de $4 \mathrm{~m}$ de profundidade e limitada a uma faixa de $10 \mathrm{~m}$ de largura. 


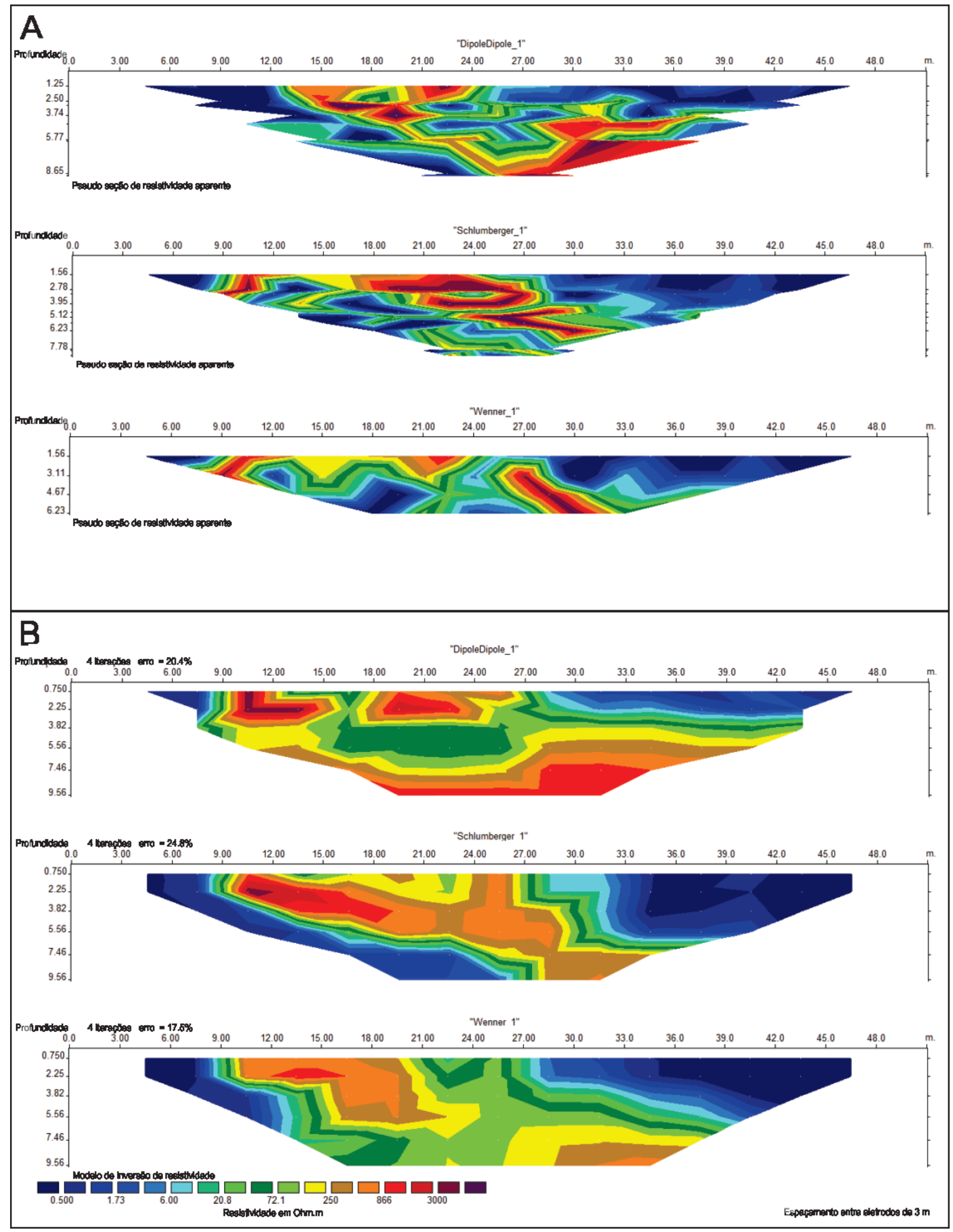

Figura 4. Modelos de inversão de resistividade para os arranjos dipolo-dipolo, Schlumberger e Wenner. A) Linha 1; B) Linha 3.

Figure 4. Inversion resistivity models for the dipole-dipole, Schlumberger and Wenner arrays lines. A) Line 1; B) Line 3. 


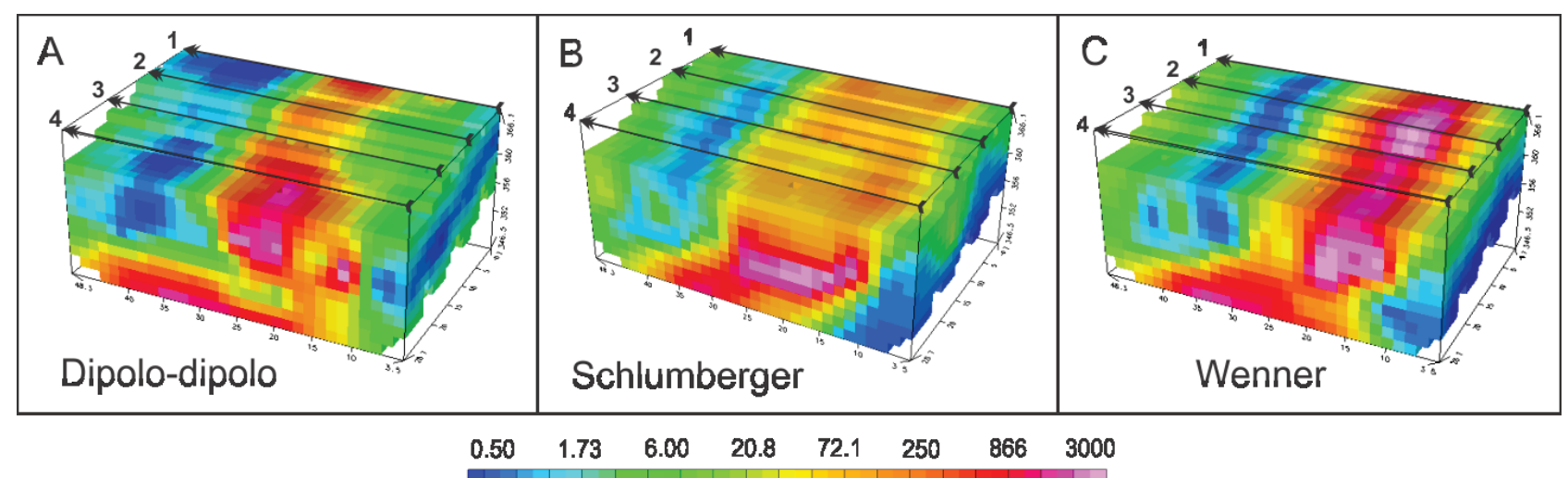

Resistividade em $\mathbf{\Omega . m}$

Figura 5. Modelos de visualização 3D em blocos resultantes da interpolação lateral dos modelos de inversão 2D. A) Arranjo dipolo-dipolo; B) Arranjo Schlumberger; C) Arranjo Wenner.

Figure 5. 3D visualization models in blocks results of lateral interpolation of 2D inversion models. A) Dipole-dipole array; B) Schlumberger array; C) Wenner array.

\section{Discussão}

A identificação de zonas de baixa resistividade é algo bastante relevante aos objetivos de estudo no contexto geológico local, visto que pode refletir a existência de zonas ou faixas de granito fraturado com percolação de águas subterrâneas.

Em outro aspecto, características intrínsecas em termos de propagação de campo e potencial elétrico produzido por diferenças de geometria em cada arranjo de aquisição de dados geoelétricos, podem justificar as diversas nuances no formato e intensidade de valores em cada zona, além possibilitar uma definição do arranjo mais adequado à definição de alvos promissores para a perfuração de poços em sistema aquífero fraturado.

Numa tentativa de evidenciar potenciais estruturas de fluxo de água subterrânea foram gerados modelos 3D de superfícies de resistividade no valor de $70 \Omega$.m para os três arranjos em estudo (Fig. 6A, 6B e 6C).

$\mathrm{O}$ arranjo dipolo-dipolo é caracterizado pela separação crescente entre eletrodos ou dipolos de corrente e potencial, ou seja, quanto maior a distância entre dipolos, maior a profundidade de investigação e menor será a intensidade de campo potencial para o caso de intensidade de corrente elétrica fixa (Mussett \& Khan, 2000). Diversos trabalhos demostram sua aplicabilidade no estudo de contaminações orgânicas e inorgânicas em condições de zona saturada, comprovadas por análises químicas (Moreira et al., 2006; Arango-Galván et al, 2011; BelmonteJiménez et al., 2012; Delgado-Rodríguez et al., 2014).

No trabalho de Nyquist et al. (2007), os autores apontam por meio de seções 2D a baixa sensibilidade do arranjo dipolo-dipolo na detecção de uma zona de falha verticalizada preenchida por água existente no maciço comprovada por meio desondagem. Este arranjo foi bastante susceptível a ruídos em estudo de detalhe realizado por Furman et al. (2003), devido a sua alta sensibilidade em níveis rasos, onde foram adotados espaçamentos de $1 \mathrm{~m}$ entre eletrodos.

Uma característica deste arranjo é a distorção lateral na propagação de campo e potencial em pequenas separações de eletrodos (investigações rasas). Esta tendência é atenuada à medida que aumenta o espaçamento entre dipolos, embora ocorra de forma concomitante uma redução na sensibilidade ou razão sinal/ ruído (investigações profundas). Neste sentido, algo inerente a fluxo dos campos elétrico e potencial é a propagação em vetor principal com ângulo próximo a $45^{\circ}$.

O modelo de isosuperficie de resistividade para o arranjo dipolo-dipolo descreve dois corpos de baixa resistividade, ambos com formato tubular e contínuo ao longo das quatro linhas tomográficas, com orientação E/W e inclinação que acompanha o relevo. O corpo de maior expressão está posicionado na porção 
final das seções, o segundo corpo, parcialmente segmentado e de menor expressão, está posicionado no inicio das seções (Fig. 6A, 6B e $6 \mathrm{C})$.

Os arranjos Schlumberger e Wenner são caracterizados por eletrodos de corrente posicionados nos extremos do dispositivo, com eletrodos de potencial fixados na posição central. O arranjo Schlumberger possibilita leituras crescentes e diversas dos eletrodos de corrente para uma mesma abertura de eletrodos de potencial, ao passo que no arranjo Wenner são necessárias aberturas crescentes em todos os eletrodos a cada leitura (Mussett \& Khan, 2000). Nos trabalhos de Furman et al. (2003) e Okpoli (2013) são descritos a maior sensibilidade destes arranjos para alvos profundos em relação do arranjo dipolo-dipolo.

O arranjo Wenner apresenta uma propagação verticalizada dos vetores de campo, ou seja, horizontalização das isosuperficies de campo elétrico e potencial, levemente atenuada em maiores espaçamentos entre eletrodos. Neste caso, ocorre o realce de estratos horizontais e sua aplicação é altamente recomendada em investigações estratigráficas e em terrenos sedimentares (Dahlin, 2000; Corwin \& Lesch, 2003; Samouëlian et al., 2003).
O modelo de isosuperficies de resistividade para o arranjo Wenner também descreve dois corpos de baixa resistividade, ambos com formato tubular e contínuo ao longo das quatro linhas tomográficas, com orientação E/W e inclinação que acompanha o relevo. Contudo, neste caso, ambos os corpos possuem dimensões equivalentes e, portanto, o corpo de baixa resistividade do início das linhas é comparativamente maior em relação ao arranjo dipolo-dipolo (Fig.6A, 6B e 6C).

O arranjo Schlumberger é caracterizado pela propagação de campo potencial semelhante a uma gota, com vetor de fluxo principal que tende a verticalização com o aumento da profundidade de investigação e distorção incipiente. Este arranjo é bastante aplicado em estudos hidrogeológicos (Salem, 2001; Sequeira Gómez \& Escolero Fuentes, 2010; Dena et al., 2012; Urrutia-Fucugauchi, 2014).

Omodelodeisosuperficies deresistividade para o arranjo Schlumberger em contraste com os demais, apresenta um corpo e uma superfície inclinada de baixa resistividade, contínuos ao longo das quatro linhas tomográficas, com direção E/W e inclinação da superfície no sentido norte. O corpo de baixa resistividade apresenta formato basicamente semelhante ao dos demais arranjos, principalmente quando analisado em perspectiva lateral (Fig. 6A, 6B e 6C).

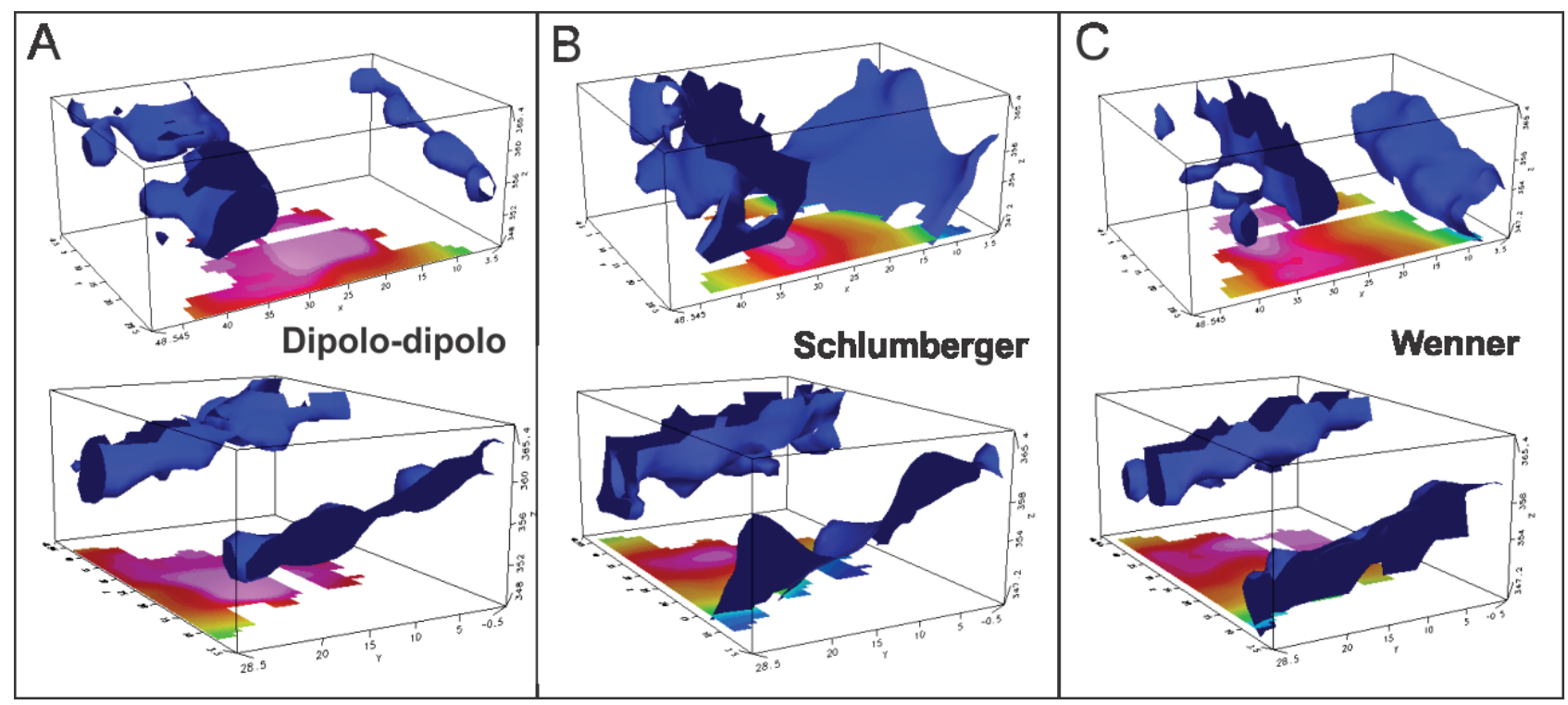

Figura 6. Modelos de isosuperficie de resistividade de $70 \Omega . \mathrm{m}$ em perspectiva frontal e lateral. A) Arranjo dipolo-dipolo; B) Arranjo Schlumberger; C) Arranjo Wenner.

Figure 6. Isosurface resistivity models of $70 \Omega . \mathrm{m}$ in frontal and lateral perspective. A) Dipole-dipole array; B) Schlumberger array; C)Wenner array. 
A superfície de baixa resistividade mostra ondulações ou irregularidades de formato, mas segue a tendência de orientação aproximada $\mathrm{N} 261 / 41^{\circ}$. Os resultados da análise estrutural no maciço granítico revelaram a existência de grupo de fraturas com orientação média N272/43․ Desta forma, a superfície de baixa resistividade reconhecida no modelo 3D para o arranjo Schlumberger deve ser indicativo de um plano de fratura saturado em água.

Aparentemente, o formato de propagação do campo elétrico numa interface de baixa resistividade com mergulho médio de $40^{\circ}$ foi adequado a uma forte refração e geração de campo potencial de baixa intensidade, de forma contínua e em diversas profundidades, algo que possibilitou a interpolação dos dados e o modelamento de uma superfície de baixa resistividade que basicamente reflete uma estrutura geológica reconhecida na área. Em contrapartida, o corpo tubular de baixa resistividade existente no final das linhas tomográficas e recorrente nos modelos dos três arranjos, deve refletir um conjunto de fraturas verticais saturadas em água, que também foram estruturas descritas no maciço granítico.

o cruzamento entre dados estruturais e uma vista em perspectiva lateral do bloco de isosuperficie 3D para o arranjo Schlumberger possibilita a projeção de planos de fratura saturados em água, para profundidades superiores àquela alcançada pelas linhas tomográficas, cujo cruzamento define um local potencialmente promissor para perfuração de poço para captação de água em sistema aquífero fraturado (Fig. 7A e 7B).

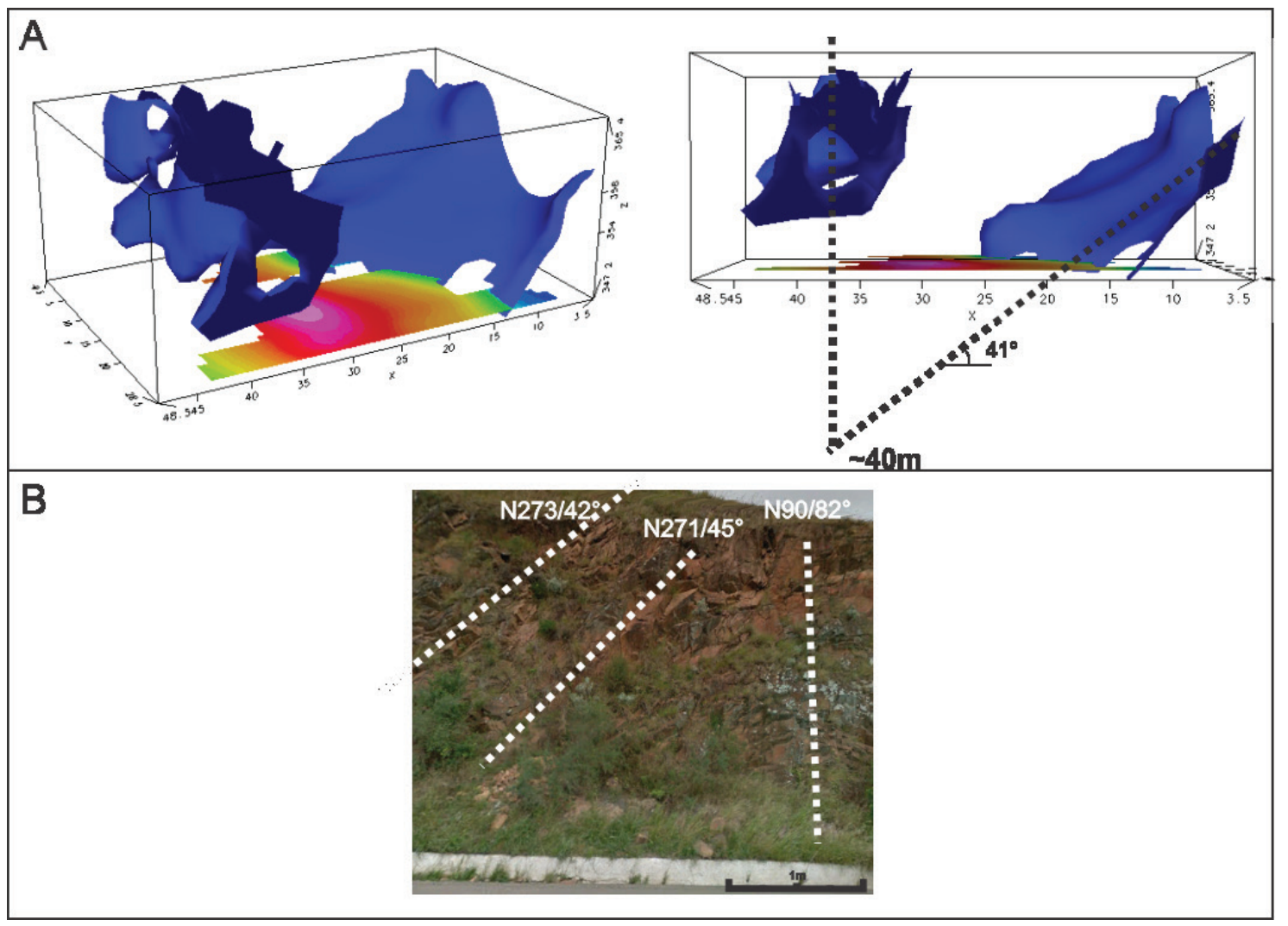

Figura 7. Modelo geofísico-estrutural. A) Vista em perspectiva e lateral com projeção de planos de fratura para isosuperficie

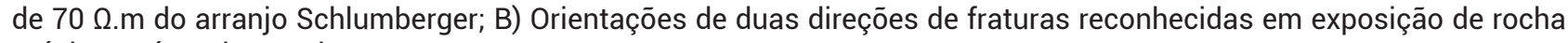
próximo a área de estudo.

Figure 7. Geophysical-structural model. A) View in perspective and lateral with projection of fractures planes for isosurface of the $70 \Omega$.m of Schlumberger array; B) Orientation of two directions of the fractures descripts in rocks exposition next to study area. 


\section{Conclusões}

A análise comparativa de modelos de inversão 2D para os arranjos dipolo-dipolo, Wenner e Schlumberger, obtidas a partir de ensaios de tomografia elétrica em área de ocorrência de granitos fraturados entre 100 m a 300 m da área de estudo, permitiram o reconhecimento de zonas com baixos valores de resistividade elétrica.

Os modelos para o arranjo dipolo-dipolo indicam uma zona restrita no início das seções, além de uma segunda zona pouco profunda entre a posição central e final das seções. Os modelos de inversão para os arranjos Wenner e Schlumberger apresentaram zonas de baixa resistividade em áreas semelhantes, mas com profundidade e continuidade lateral muito mais expressivas. Em todos os casos há um acentuado contraste de valores de resistividade: elevados valores são indicativos de áreas com granito inalterado, enquanto os muito baixos são relacionados a solo/saprolito argiloso saturado.

$\mathrm{Na}$ interface destas áreas com extremos de resistividade elétrica foram ajustadas isosuperfícies de resistividade elétrica, a partir da interpolação lateral dos modelos de inversão 2D, que resultaram em modelos de visualização 3D (quasi-3D), numa tentativa de delineamento de estruturas de fluxo em aquífero fraturado.

Os três arranjos revelaram sensibilidade no reconhecimento de um provável de um conjunto de estruturas verticais saturadas em água, cujo modelamento da interface externa revela uma estrutura tubular, rasa e contínua na direção N81 ${ }^{\circ}$ em posição transversal e no início das linhas. Em exposição de granitos a poucas dezenas de metros da área de estudo, foram reconhecidos intervalos com sistemas de fraturas verticais e com evidência de umidade, limitados por zonas de granito maciço e inalterado.

Os resultados demostram que a despeito do contraste de propriedades elétricas existentes entre os alvos estudados e o ambiente em redor, em alguns casos não houve o reconhecimento da estrutura por meio de determinado arranjo.

A versatilidade do arranjo Dipolodipolo em termos de logística de aquisição de dados torna seu uso atrativo em aquisições de campo. A sensibilidade do arranjo Wenner no estudo de sistemas geológicos sedimentares e estratificados está baseada na forma horizontalizada de propagação de frentes de campo elétrico e potencial. Contudo, os resultados de aplicação destes arranjos possibilitaram o modelamento parcial de estruturas de fluxo em sistema fraturado.

Somente o arranjo Schlumberger demonstrou sensibilidade e proporcionou dados satisfatórios para identificação de dois sistemas de fraturas com orientações distintas, bastante semelhantes ao descrito em exposições de rocha próximas a área de estudos. A projeção e intersecção destas duas famílias de fratura indica uma área altamente promissora para perfuração e captação subterrânea. A cobertura de solo local torna praticamente impossível a análise estrutural direta e uma determinação precisa de locais com contexto hidrogeológico favorável.

Os resultados sugerem a aplicação de estudos integrados e a realização de ensaios geofísicos preliminares em áreas de alta complexidade geológica, particularmente quando aplicados ensaios de tomografia elétrica em pesquisas de aquíferos fraturados. Investigações a partir de arranjos geoelétricos consagrados podem resultar em dados inconclusivos em estudos hidrogeológicos, induzir a seleção de locais inapropriados e resultar em poços secos, equívocos que colocam em descrédito a geofísica como ferramenta para locação de poços profundos.

\section{Referências}

Aizebeokhai, A P., Olayinka, A.I., Singh, V.S. \& Uhuegbu, C.C. 2011. Effectiveness of3D geoelectrical resistivity imaging using parallel 2D profiles. International Journal of the Physical Sciences, 6: 5623-5647.

Arango-Galván, C., Torre-González, B., ChávezSegura, R.E., Tejero-Andrade, A., CifuentesNava, G. \& Hernández-Quintero, E. 2011. Structural pattern of subsidence in an urban area of the southeastern Mexico Basin inferred from electrical resistivity tomography. Geofísica Internacional, 50: 401-409.

Arango-Galván, C., Flores-Márquez, E.L., 
Hernández-Espriú, J.A., Arias-Paz, A. \& Sagahón-López, E.J. 2016. Shallow geoelectrical characterization of a small portion of the Basin of Mexico aquifer: Towards a better resource management. Geofísica Internacional, 55(4): 215-225.

Belmonte-Jiménez, S., Jimenez-Castañeda, M.E., Pérez-Flores, M.A., Campos-Enríquez J., Reyes-López, J.A. \& Salazar-Peña, L. 2012. Characterization of a leachate contaminated site integrating geophysical and hydrogeological information. Geofísica Internacional, 51(3): 309-321.

Bittencourt, M.F. 1983. Metamorfitos da região de Caçapava do Sul, RS - Geologia e relações com o corpo granítico. In: SIMPÓSIO SULBRASILEIRO DE GEOLOGIA, 1, 1983, Porto Alegre. Anais... Porto Alegre, SBG, v. 1 p. 37-49.

Chambers, J.E., Kuras, O., Meldrum, P. I., Ogilvy, R. D. \& Hollands, J. 2006. Electrical resistivity tomography applied to geologic, hydrogeologic, and engineering investigations at a former waste-disposal site. Geophysics, 71: 231-239.

Côrtes, A.R.P., Moreira, C.A., Veloso, D.I.K., Vieira, L.B. \& Bergonzoni, F.A. 2016. Geoelectrical prospecting for a copper-sulfide mineralization in the Camaquã sedimentary basin, Southern Brazil. Geofísica Internacional, 55(3): 107-117.

Corwin, D.L. \& Lesch, S.M. 2003. Application of soil electrical conductivity to precision agriculture: theory, principles, and guidelines. Agronomy Journal, 95: 455-471.

CPRM. Companhia de Pesquisas de Recursos Minerais. 2000. Folha Cachoeira do Sul, RS. Escala 1:250.000.

Dahlin, T. 2000. Short note on electrode chargeup effects in DC resistivity data acquisition using multi-electrode arrays. Geophysical Prospecting, 48: 181-187.

Delgado-Rodríguez, O, Flores-Hernández, D, Amezcua-Allieri, M.A, Shevnin, V, RosasMolina, A \& Marín-Córdova, S. 2014. Joint interpretation of geoelectrical and volatile organic compounds data: a case study in a hydrocarbons contaminated urban site. Geofísica Internacional, 53,183-198.

Dena, O.S., Griselda Obeso, C., Doser, D., Leyva, J.E., Rascon, E., Gómez, F. \& Domínguez, M.A.
2012. Using subsurface geophysical methods in flood control: A resistivity survey to define underground storage capacity of a sand body in Ciudad Juárez, Mexico. Geofísica Internacional, 51: 225-249.

EMBRAPA. Empresa Brasileira de Pesquisa Agropecuária. 2018. Sistema Brasileiro de Classificação de Solos. Brasília, EMBRAPA, $356 \mathrm{p}$.

Furman, A., Ferre, T.P.A. \& Warrick, A.W. 2003. A sensitivity analysis of electrical resistivity tomography array types using analytical element modeling. Vadose Zone Journal, 2: 416-423.

Gandolfo, O.C.B. \& Gallas, J.D.F. 2005. Eletrorresistividade 3D: uma avaliação preliminar da técnica. Revista Brasileira de Geofísica, 23 (2), p. 191-198.

Griffiths, D.H. \& Barker, R.D. 1993. Two dimensional resistivity imaging and modeling in areas of complex geology. Journal of Applied Geophysics, 29(3):211-226

Helene, L.P.I., Moreira, C.A. \& Carrazza, L.P. 2016. Applied geophysics on a soil contaminated site by chromium of a tannery in Motuca (SP, Brazil). Revista Brasileira de Geofísica, 34(3): 309-317.

Knödel, K., Lange, G. \& Voigt, H. 2007. Environmental geology: handbook of fields methods and case studies. Hannover, Springer, $1374 \mathrm{p}$.

Loke, M.H. \& Barker, R.D. 1996. Rapid LeastSquares Inversion of Apparent Resistivity Pseudosections Using a Quasi-Newton Method. Geophysical Prospecting 44(1):131 152

Milsom, J.J. \& Eriksen, A. 2011. Field Geophysics. Chichester, John Wiley \& Sons Ltd, 279p.

Moreira, C.A., Dourado, J.C. \& Braga, A.C.O. 2006. Aplicação da técnica de caminhamento elétrico em área contaminada por derivados de petróleo. Revista Brasileira de Geofísica, 24, 383-392.

Moreira, C.A., Lapola, M.M. \& Carrara, A. 2016. Comparative analyzes among electrical resistivity tomography arrangements in the characterization of flow structure in free aquifer. Geofísica Internacional, 55(2): 119129. 
Moreira, C.A., Lopes, S.M., Schweig, C. \& Seixas A.R. 2012. Geoelectrical prospection of disseminated sulfide mineral occurrences in Camaquã sedimentary basin, Rio Grande do Sul state, Brazil. Revista Brasileira de Geofísica, 30: 169-179.

Moreira, C.A., Helene, L.P.I. \& Côrtez, A.R.P. 2017. $D C$ resistivity method applied in the monitoring of diesel leakage in a railway accident in São Manuel city, São Paulo state (Brazil). Revista Brasileira de Geofísica, 30(1): 5-14.

Moreira, C.A., Lopes, S.M., Schweig, C. \&Seixas A.R. 2012. Geoelectrical prospection of disseminated sulfide mineral occurrences in Camaquã sedimentary basin, Rio Grande do Sul state, Brazil. Revista Brasileira de Geofísica, 30: 169-179.

Moon, C.J., Whateley, M.K.G. \&Evans, A.M. 2006. Introduction of mineral exploration. $2^{\circ}$ ed., Malden, Blackwell Publishing, 499p.

Mussett, A.E. \& Khan, M.A. 2000. Looking into the Earth: an introduction to geological geophysics. New York, Cambridge University Press, 493p.

Nyquist, J.E., Peake, J.S. \& Roth, M. J.S. 2007. Comparison of an optimized resistivity array with dipole-dipole soundings in karst terrain. Geophysics, 72: 139-144.

Orellana, E. 1972. Prospeccion Geoelectrica en Corriente Continua, Biblioteca Técnica Philips, Madrid, Paraninfo, 523p.

Okpoli, C.C. 2013. Sensitivity and resolution capacity of Electrode configurations. International Journal of Geophysics, 2013, 12 p. p.

Rubin, Y. \& Hubbard, S.S. 2005. Hydrogeophysics. Dordrecht, Springer, 527p.

Salem, H.S. 2001. Modelling of lithology and hydraulic conductivity of shallow sediments from resistivity measurements using Schlumberger vertical electrical soundings. Energy Sources, 23: 599-618.
Samouëlian, A., Cousin, I., Richard, G., Tabbagh, A. \& Bruand, A. 2003. Electrical Resistivity Imaging for Detecting Soil Cracking at the Centimetric Scale. Soil Science Society of America Journal, 67: 1319-1326.

Sara, M. 2003. Site assessment and remediation handbook. $2^{\circ}$ ed., Lewis Publishers, Florida, $1161 p$.

Sequeira Gómez, L. \& Escolero Fuentes, O. 2010. The application of electrical methods in exploration for ground water resources in the River Malacatoya sub-basin, Nicaragua. Geofísica Internacional, 49: 27-41.

Telford, W.M., Geldart, L.P. \& Sheriff R.E. 2004. Applied Geophysics. New York, Cambridge University Press, 774p.

Urrutia-Fucugauchi, J., Trigo-Huesca, A., TéllezGarcía, E., Pérez-Cruz, L. \& Méndez-Rivero F. 2014. Volcano-sedimentary stratigraphy in the Valsequillo Basin, Central Mexico inferred from electrical resistivity soundings. Geofísica Internacional, 53: 87-94.

Vieira, L.B., Moreira C.A., Côrtes A.R.P. \& Luvizotto, G.L. 2016. Geophysical modeling of the manganese deposit for Induced Polarization method in Itapira (Brazil). Geofísica Internacional, 55(2): 107-117.

Zhou, Q.Y., Shimada, J. \& Sato, A., 2001. Threedimensional spatial and temporal monitoring of soil water content using electrical resistivity tomography. Water Resources Research, 37: 273-285. 